\title{
State-Dependent Strength-of-Preference
}

\author{
Yutaka Nakamura \\ Graduate School of Systems and Information Engineering \\ Division of Social Systems and Management \\ University of Tsukuba \\ 1-1-1 Tennnoudai, Tsukuba, Ibaraki 305-8573, Japan
}

June 9, 2015

\begin{abstract}
When a utility function is state-dependent, ex ante preferences for acts cannot elicit unique subjective probabilities without auxiliary devices. This paper introduces expost strength-of-preferences for state-contingent decision outcomes. Then we prove that a consistency condition between ex ante preference and ex post strength-of-preference relations can separate utility and unique subjective state probabilities.
\end{abstract}

Keywords: state-dependent utility, strength-of-preference, ex ante-ex post consistency, unique subjective probability

JEL Classification: D81 


\section{Introduction}

In the subjective expected utility (SEU) paradigm, subjective state probabilities are elicited through ex ante preferences (i.e., preferences before uncertainties are resolved) for acts, which are mappings from the state space into decision outcomes. Savage (1954) related subjective likelihood judgments to ex ante preferences to drive unique subjective state probabilities. On the other hand, Anscombe and Aumann (1963) obtained unique subjective state probabilities as scaling constants. Since preferences are assumed to be state-independent, those scaling constants turn out to represent induced likelihood judgements as in Savage's framework. However, when preferences are state-dependent, their approaches do not work to elicit unique subjective state probabilities.

It has been argued that separability of utility and subjective state probabilities may be of no significance when utility is state-dependent. For identifying unique probability in the literature, however, several exogenous assumptions are devised to invoke information other than observable choice (e.g., see Wakker and Zank, 1999, and Drèze and Rustichini, 2004, for detailed surveys and discussion). Drèze and Rustichini grouped approaches for the identification into three categories: hypothetical preferences, moral hazard, and (event) conditional preferences. The first two categories are concerned with ex ante preferences, while the last assumes that the decision maker has preferences for acts conditional on different events (or states).

This paper proposes another attempt to separate utility and subjective probability. Unlike those approaches, we shall allow for two primitives for the decision maker's subjective judgments. In addition to ex ante preferences, we introduce ex post strength-of-preferences for state-contingent decision outcomes. Although state-conditional preferences are induced by ex post strength-of-preferences, we do not require that the decision maker has ex post preferences for outcomes in "different" states. Instead, we shall assume that strength-ofpreferences at one state can be introspectively comparable to strength-of-preferences at other state.

While the notion of strength-of-preference has been criticized in the literature because of its unobservable nature of judgments, there have been many attempts to operationalize in decision analysis (e.g., see von Winterfeldt and Edwards, 1986, and Farquhar and Keller, 1989 for discussions of measurement methodologies, and Butler et. al., 2014 for a recent experimental study). To the best of my knowledge, Sarin (1982) was the only attempt to extend the domain of strength-of-preferences to decision making under uncertainty. He showed conditions under which a (state-independent) utility function in a SEU framework with finite states can be legitimately interpreted to measure "ex ante" strength-of-preferences between acts, including decision outcomes identified with constant acts.

Observe that our application of strength-of-preferences in the SEU framework is quite different from Sarin's. We apply two well known utility representations of ex ante preferences and ex post strength-of-preferences in our set-up. The main contribution of the paper is to discover how those two representations are consistently combined to obtain unique subjective probabilities as scaling constants.

A legitimacy of interpreting those constants as subjective probabilities may be demon- 
strated by an example. Suppose that the decision maker is concerned with her health states, bad or good health, and her final wealth levels. If she will not make any action now, she knows that her wealth level will be $w_{b}$ if her health will become bad, and $w_{g}$ otherwise. Now she is offered to select one of two acts: one is to make her wealth increased by amount $\delta_{b}$ if her health turns out to be bad, and otherwise unchanged from no action, and another is the other way around from the former act, i.e., to increase wealth by amount $\delta_{g}$ if her health will be good, and otherwise unchanged from no action. Suppose that preference intensity for a situation in which the decision maker's wealth $w_{b}$ at bad health can be increased by amount $\delta_{b}$ is judged to be equal to preference intensity for a situation in which her wealth $w_{g}$ at healthy state can be increased by amount $\delta_{g}$. Then if she judges that the two acts are indifferent, then it may be natural to infer that her likelihood judgement about the occurrence of each health state would be equally likely. Of course, if she prefers the former act to the latter, we may conclude that bad health is judged to be more likely than good health.

The paper is organized as follows. In Section 2, we introduce two axiom systems: one is for ex ante preferences and another for ex post strength-of-preferences. Section 3 presents ex ante-ex post consistency axiom and the main representation theorem which combines two utility representations of ex ante preferences and ex post strength-of-preferences to yield state dependent subjective expected utility. Section 4 concludes the paper.

\section{Ex Ante and Ex Post Preferences}

Let $S=\left\{s_{1}, \ldots, s_{n}\right\}$ be a finite set of states. Exactly one state is true and other states are false. The decision maker does not know ex ante which state is true. An act is a function from $S$ into the real line $\mathbb{R}$. Let $X$ be a set of acts for which, for every $s_{i} \in S$, $X_{i}=\left\{f\left(s_{i}\right): f \in X\right\}$ is a real interval. Thus $X$ can be identified with a rectangular subset $X_{1} \times \cdots \times X_{n}$ of $\mathbb{R}^{n}$ through the mappings $f \rightarrow\left(f\left(s_{1}\right), \ldots, f\left(s_{n}\right)\right)$. Therefore, an act will be identified with a real vector $\boldsymbol{x} \in \mathbb{R}^{n}$, bold faced letters, whose $i$-component will be $x_{i} \in X_{i}$, i.e., $\boldsymbol{x}=\left(x_{1}, \ldots, x_{n}\right)$. For any act $\boldsymbol{x} \in X$, an act whose $i$-component $x_{i}$ is replaced by some $\alpha_{i} \in X_{i}$ will be denoted by $\alpha_{i} \boldsymbol{x}$. In what follows, we shall identify $N=\{1, \ldots, n\}$ as the set $S$ of states.

Let $\succsim$ denote the ex ante preference relation on $X$, i.e., for $\boldsymbol{x}, \boldsymbol{y} \in X, \boldsymbol{x} \succsim \boldsymbol{y}$ means that the decision maker weakly prefers $\boldsymbol{x}$ to $\boldsymbol{y}$ before she knows the true state. The indifference and strict preference relations, denoted respectively $\sim$ and $\succ$, are defined in the usual way: $\boldsymbol{x} \sim \boldsymbol{y}$ if $\boldsymbol{x} \succsim \boldsymbol{y}$ and $\boldsymbol{y} \succsim \boldsymbol{x} ; \boldsymbol{x} \succ \boldsymbol{y}$ if $\neg(\boldsymbol{y} \succsim \boldsymbol{x})$. State $i \in N$ is said to be null if $a_{i} \boldsymbol{x} \sim \boldsymbol{x}$ for all $a_{i} \in X_{i}$ and all $\boldsymbol{x} \in X$. Let $N^{*}$ denote the set of all nonull states. Strict monotonicity of $\succsim$ is said to hold if $a_{i} \boldsymbol{x} \succ b_{i} \boldsymbol{x}$ for all nonnull state $i$ and all $\boldsymbol{x} \in X$ and all $a_{i}, b_{i} \in X_{i}$ whenever $a_{i}>b_{i}$. Then we assume that the following three axioms hold for the ex ante preference relation $\succsim$ on $X$ :

Axiom EA1. $\succsim$ is a weak order.

Axiom EA2. For all $\boldsymbol{y} \in X,\{\boldsymbol{x} \in X: \boldsymbol{x} \succsim \boldsymbol{y}\}$ and $\{\boldsymbol{x} \in X: \boldsymbol{y} \succsim \boldsymbol{x}\}$ are closed in $X$. Axiom EA3. $\succsim$ is strictly monotonic. 
It is well known (see Debreu, [4]) that Axioms EA1 and EA2 hold if and only if there exists a continuous real valued function $V$ on $X$ such that, for all $\boldsymbol{x}, \boldsymbol{y} \in X$,

$$
\boldsymbol{x} \succsim \boldsymbol{y} \Longleftrightarrow V(\boldsymbol{x}) \geq V(\boldsymbol{y})
$$

By axiom EA3, $V$ is strictly monotonic in the sense that for nonnull $i, V\left(a_{i} \boldsymbol{x}\right)>V\left(b_{i} \boldsymbol{x}\right)$ whenever $a_{i}>b_{i}$, and, for null $i, V\left(a_{i} \boldsymbol{x}\right)=V\left(b_{i} \boldsymbol{x}\right)$ if $\boldsymbol{x} \in X$ and $a_{i}, b_{i} \in X_{i}$.

In what follows, if there is an $\alpha \in \mathbb{R}$ such that $\alpha \in X_{i} \cap X_{j}$ for distinct $i$ and $j$, then $\alpha \in X_{i}$ and $\alpha \in X_{j}$ should be interpreted as distinct numbers. One way to do this may be that each $X_{i}$ is replaced by $\left\{(\alpha, i): \alpha \in X_{i}\right\}$. To avoid such notational complication, we will understand each $X_{i}$ as a set of real numbers that are state-contingent to $i$. Let ${ }^{*}$ be the $e x$ post strength-of-preference relation which is defined on the set of all pairs of numbers from any one of product sets $X_{i} \times X_{i}$ for all nonnull $i \in N$, i.e., on the set

$$
\bigcup_{i \in N^{*}} X_{i} \times X_{i}
$$

Thus, for $\left(x_{i}, y_{i}\right) \in X_{i} \times X_{i}$ and $\left(z_{j}, w_{j}\right) \in X_{j} \times X_{j},\left(x_{i}, y_{i}\right) \succsim^{*}\left(z_{j}, w_{j}\right)$ means that the degree of preference for $x_{i}$ over $y_{i}$ when state $i$ is true is weakly larger than the degree of preference for $z_{j}$ over $w_{j}$ when state $j$ is true. This is known as a cross-modality ordering in psychology literature (see Krantz, et. al., 1971), whose numerical representation is given as follows: there exist continuous functions $v_{i}$ on $X_{i}$ for $i \in N^{*}$ such that, for all $i, j \in N^{*}$, all $\left(x_{i}, y_{i}\right) \in X_{i} \times X_{i}$, and all $\left(z_{j}, w_{j}\right) \in X_{j} \times X_{j}$,

$$
\left(x_{i}, y_{i}\right) \succsim^{*}\left(z_{j}, w_{j}\right) \Longleftrightarrow v_{i}\left(x_{i}\right)-v_{i}\left(y_{i}\right) \geq v_{j}\left(z_{j}\right)-v_{j}\left(w_{j}\right) .
$$

Furthermore, if $u_{i}$ is another continuous function on $X_{i}$ for $i \in N^{*}$, which can replace $v_{i}$ in the representation, then there are numbers $\alpha>0$ and $\beta_{i}$ for $i \in N^{*}$ such that $u_{i}=\alpha v_{i}+\beta_{i}$ for $i \in N^{*}$. For the latter property of $v_{i}$ for $i \in N^{*}$, we say that $v_{i}$ for $i \in N^{*}$ are unique up to a similar positive linear transformation (or simply, similar PLT-unique).

The indifference and strict strength-of-preference relations, denoted respectively $\sim^{*}$ and $\succ^{*}$, are defined in the usual way: for $i \in N^{*},\left(x_{i}, y_{i}\right) \sim^{*}\left(z_{j}, w_{j}\right)$ if $\left(x_{i}, y_{i}\right) \succsim^{*}\left(z_{j}, w_{j}\right)$ and $\left(z_{j}, w_{j}\right) \succsim^{*}\left(x_{i}, y_{i}\right) ;\left(x_{i}, y_{i}\right) \succ^{*}\left(z_{j}, w_{j}\right)$ if $\neg\left(\left(z_{j}, w_{j}\right) \succsim^{*}\left(x_{i}, y_{i}\right)\right)$. We assume that the following topological version of the axioms of the cross-modality ordering holds for the ex post strength-of-preference relation $\succsim^{*}$ on $\bigcup_{i \in N^{*}} X_{i} \times X_{i}$, which are understood as applying to all $i, j \in N^{*}$, all $x_{i}, y_{i}, z_{i} \in X_{i}$, and all $x_{j}^{\prime}, y_{j}^{\prime}, z_{j}^{\prime} \in X_{j}$.

Axiom EP1. ¿* is a weak order.

Axiom EP2. If $\left(x_{i}, y_{i}\right) \succsim^{*}\left(x_{j}^{\prime}, y_{j}^{\prime}\right)$, then $\left(y_{j}^{\prime}, x_{j}^{\prime}\right) \succsim^{*}\left(y_{i}, x_{i}\right)$.

Axiom EP3. If $\left(x_{i}, y_{i}\right) \succsim^{*}\left(x_{j}^{\prime}, y_{j}^{\prime}\right)$ and $\left(y_{i}, z_{i}\right) \succsim^{*}\left(y_{j}^{\prime}, z_{j}^{\prime}\right)$, then $\left(x_{i}, z_{i}\right) \succsim^{*}\left(x_{j}^{\prime}, z_{j}^{\prime}\right)$.

Axiom EP4. $\left\{\left(a_{i}, b_{i}\right):\left(a_{i}, b_{i}\right) \succsim^{*}\left(x_{j}^{\prime}, y_{j}^{\prime}\right)\right\}$ and $\left\{\left(a_{i}, b_{i}\right):\left(x_{j}^{\prime}, y_{j}^{\prime}\right) \succsim^{*}\left(a_{i}, b_{i}\right)\right\}$ are closed in $X_{i} \times X_{i}$.

When strength-of-preference (or preference intensity) for outcome $x_{i} \in X_{i}$ over $y_{i} \in X_{i}$ is at least as large as strength-of-preference for outcome $z_{j} \in X_{j}$ over $w_{j} \in X_{j}$, the ordering 
judgment would be reversed if the roles of $x_{i}$ and $y_{i}$ in the first comparison and $z_{j}$ and $w_{j}$ in the second are exchanged. Axiom EP2 guarantees this requirement. Axiom EP3 says that comparisons of concatenated strength-of-preferences should preserve the ordering of the premised comparisons of strength-of-preferences. This clearly holds true if strength-ofpreferences are to be measured as utility differences.

The topological version is indeed stronger than an algebraic version in Krantz et. al. (1971) who proved the representation by assuming solvability and Archimedean axioms (for the definitions, see Chapter 4 in Krantz, et. al., 1971) in place of Axiom EP4. Clearly, it follows from Axioms EP1 and EP4 that there exists a continuous function $U$ on $\bigcup_{i \in N^{*}} X_{i} \times X_{i}$ such that, for all $i, j \in N^{*}$, all $\left(x_{i}, y_{i}\right) \in X_{i} \times X_{i}$, and all $\left(z_{j}, w_{j}\right) \in X_{j} \times X_{j}$,

$$
\left(x_{i}, y_{i}\right) \succsim^{*}\left(z_{j}, w_{j}\right) \Longleftrightarrow U\left(x_{i}, y_{i}\right) \geq U\left(z_{j}, w_{j}\right) .
$$

The solvability and Archimedean axioms readily follow from the continuity of $U$. Of course, the role of Axioms EP2 and EP3 is to decompose $U$ into $U\left(x_{i}, y_{i}\right)=v_{i}\left(x_{i}\right)-v_{i}\left(y_{i}\right)$ for $i \in N^{*}$.

Finally we note that monotonicity is not imposed here. It is the property induced by the consistency axiom introduced in the next section.

\section{State-Dependent Representation}

In this section, we shall assume that ex ante axioms EA1-EA3 for the ex ante preference relation $\succsim$ on $X=X_{1} \times \cdots \times X_{n}$ and ex post axioms EP1-EP4 for the ex post strengthof-preference relation $\succsim^{*}$ on $\bigcup_{i \in N^{*}} X_{i} \times X_{i}$ hold. Then there exist a strictly monotonic continuous utility function $V$ on $X$ for $\succsim$ and similar PLT-unique continuous strength-ofpreference functions $v_{i}$ on $X_{i}\left(i \in N^{*}\right)$ for $\succsim^{*}$. At this point, however, nothing can be said about any relation between $V$ and a set $\left\{v_{i}: i \in N^{*}\right\}$.

In what follows, with no loss of generality, we shall assume that $N^{*}=\{1, \ldots, m\}$ for some $2 \leq m \leq n$. Our goal is to find a condition which guarantees the existence of a unique positive probability vector $\boldsymbol{p}=\left(p_{1}, \ldots, p_{m}\right) \in \mathbb{R}^{m}$ such that $\sum_{i=1}^{m} p_{i}=1$ and, for all $\boldsymbol{x} \in X$,

$$
V(\boldsymbol{x})=\sum_{i=1}^{m} p_{i} v_{i}\left(x_{i}\right) .
$$

It follows from the representation that $v_{i}$ for $i \in N^{*}$ are strictly increasing. Furthermore, if $\left(a_{i}, x_{i}\right) \sim^{*}\left(b_{j}, x_{j}\right)$ for $i, j \in N^{*}$, then, for all $\boldsymbol{x} \in X$,

$$
a_{i} \boldsymbol{x} \succsim b_{j} \boldsymbol{x} \Longleftrightarrow p_{i} \geq p_{j} .
$$

This says that, for nonnull $i$ and $j$, if preference intensity for $a_{i}$ over $x_{i}$ is judged to be equal to preference intensity for $b_{j}$ over $x_{j}$, then state $i$ is weakly more likely than state $j$ if and only if act $a_{i} \boldsymbol{x}$ is weakly preferred to act $b_{j} \boldsymbol{x}$. 
A consistency condition between ex ante preference and ex post strength-of-preference relations that we need is stated as follows, where the axiom is understood as applying to all $\boldsymbol{x}, \boldsymbol{y}, \boldsymbol{z}, \boldsymbol{w} \in X$.

Ex ante-Ex post Consistency Axiom: If $\boldsymbol{x} \succsim \boldsymbol{y}$ and $\left(z_{i}, w_{i}\right) \succsim^{*}\left(x_{i}, y_{i}\right)$ for some nonnull $i \in N^{*}$, then $z_{i} \boldsymbol{x} \succsim w_{i} \boldsymbol{y}$. If, in addition, $\left(z_{i}, w_{i}\right) \succ^{*}\left(x_{i}, y_{i}\right)$, then $z_{i} \boldsymbol{x} \succ w_{i} \boldsymbol{y}$.

This axiom says that if, for some nonnull $i$, preference intensity for outcome $z_{i}$ over outcome $w_{i}$ is larger than or equal to preference intensity for outcome $x_{i}$ over outcome $y_{i}$, then act $z_{i} \boldsymbol{x}$ should be weakly preferred to act $w_{i} \boldsymbol{y}$ whenever act $\boldsymbol{x}$ is weakly preferred to act $\boldsymbol{y}$. If, in addition, the comparison of those preference intensities is strict, then act $z_{i} \boldsymbol{x}$ should be preferred to act $w_{i} \boldsymbol{y}$. This implies that ex post strength-of-preferences monotonically induce ex ante preferences. Since ex ante preferences are observable by choices, it would be desirable if they are induced by ex post strength-of-preferences. This is partially possible, i.e., ex post strength-of-preferences for the same state can be observed by ex ante preferences. To see this, we assume that, for $i \in N^{*}, a_{i} \boldsymbol{x} \succsim b_{i} \boldsymbol{y}$ and $d_{i} \boldsymbol{x} \succsim c_{i} \boldsymbol{y}$. If $\left(c_{i}, d_{i}\right) \succ^{*}\left(a_{i}, b_{i}\right)$, then, by the consistency axiom, $c_{i} \boldsymbol{x} \succ d_{i} \boldsymbol{y}$, a contradiction. Hence we conclude that $\left(a_{i}, b_{i}\right) \succsim^{*}\left(c_{i}, d_{i}\right)$. When one of $a_{i} \boldsymbol{x} \succsim b_{i} \boldsymbol{y}$ and $d_{i} \boldsymbol{x} \succsim c_{i} \boldsymbol{y}$ is strict, the consistency axiom together with axiom EP2 implies that $\left(a_{i}, b_{i}\right) \succ^{*}\left(c_{i}, d_{i}\right)$. Unfortunately, however, there seems to be no way to infer ex post strength-of-preferences for distinct states from ex ante preferences.

An easy implication of the consistency axiom together with monotonicity axiom EA3 is that, for all $i \in N^{*}, v_{i}$ is strictly increasing. To see this, assume that, for $i \in N^{*}, a_{i}>b_{i}$ and $\left(b_{i}, a_{i}\right) \succsim^{*}\left(x_{i}, x_{i}\right)$. Then, by the axiom, $b_{i} \boldsymbol{x} \succsim a_{i} \boldsymbol{x}$. By axiom EA3, $a_{i}>b_{i}$ implies that $a_{i} \boldsymbol{x} \succ b_{i} \boldsymbol{x}$. This is a contradiction. Hence $a_{i}>b_{i}$ implies that $\left(a_{i}, b_{i}\right) \succ^{*}\left(x_{i}, x_{i}\right)$. That is, $v_{i}$ is strictly increasing.

The representational implication of the ex ante-ex post consistency axiom is given by the following main theorem.

The Main Theorem. Suppose that $N^{*}=\{1, \ldots, m\} \subset N$ is the set of nonnull states with $m \geq 2$. Then the ex ante-ex post consistency axiom holds if and only if there exists a unique positive probability vector $\boldsymbol{p} \in \mathbb{R}^{m}$ such that, for all $\boldsymbol{x}, \boldsymbol{y} \in X$,

$$
\boldsymbol{x} \succsim \boldsymbol{y} \Longleftrightarrow \sum_{i=1}^{m} p_{i} v_{i}\left(x_{i}\right) \geq \sum_{i=1}^{m} p_{i} v_{i}\left(y_{i}\right)
$$

Proof. The necessity of ex ante-ex post consistency easily follows. Thus we show its sufficiency. In what follows, we assume that ex ante-ex post consistency holds. First we note that ex ante preference relation $\succsim$ satisfies the following condition, understood as applying to all $i \in N$, all $x_{i}, y_{i}, z_{i}, w_{i} \in X_{i}$, and all $\boldsymbol{a}, \boldsymbol{b}, \boldsymbol{c}, \boldsymbol{d} \in X$ :

Generalized triple cancellation: If $x_{i} \mathbf{a} \succsim y_{i} \mathbf{b}, z_{i} \mathbf{b} \succsim w_{i} \mathbf{a}$, and $y_{i} \mathbf{c} \succsim x_{i} \mathbf{d}$, then $z_{i} \mathbf{c} \succsim w_{i} \mathbf{d}$.

To prove this, we first assume that $i$ is null. Since $y_{i} \mathbf{c} \succsim x_{i} \mathbf{d}$, it follows from the definition of nullity and axiom EA1 that $z_{i} \mathbf{c} \succsim w_{i} \mathbf{d}$. Next, we assume that $i \in N^{*}$ and $\left(w_{i}, z_{i}\right) \succ^{*}\left(x_{i}, y_{i}\right)$. 
Since $x_{i} \mathbf{a} \succsim y_{i} \mathbf{b}$, the consistency axiom implies that $w_{i} \mathbf{a} \succ z_{i} \mathbf{b}$, a contradiction. Thus $\left(x_{i}, y_{i}\right) \succsim^{*}\left(w_{i}, z_{i}\right)$. By axiom EP2, $\left(z_{i}, w_{i}\right) \succsim^{*}\left(y_{i}, x_{i}\right)$. By the consistency axiom, $z_{i} \mathbf{c} \succsim w_{i} \mathbf{d}$.

It was proved by Wakker (1989) that, by axioms EA1 and EA2 together with generalized triple cancellation, there are similar PLT unique continuous real valued functions $f_{i}$ on $X_{i}$ for $i=1, \ldots, n$ such that, for all $\boldsymbol{x}, \boldsymbol{y} \in X$,

$$
\boldsymbol{x} \succsim \boldsymbol{y} \Longleftrightarrow \sum_{i=1}^{n} f_{i}\left(x_{i}\right) \geq \sum_{i=1}^{n} f_{i}\left(y_{i}\right) .
$$

Furthermore, $f_{i}$ is constant if $i$ is null. By axiom EA3, $f_{i}$ is strictly increasing if $i$ is nonnull.

In what follows, for $i \in N$ and all $\boldsymbol{x}, \boldsymbol{y} \in X$, define $\phi_{i}(\boldsymbol{x}, \boldsymbol{y})=\sum_{j \neq i}\left(f_{j}\left(x_{j}\right)-f_{j}\left(y_{j}\right)\right)$. First we prove that if $i \in N$ is nonnull, then, for all $\left(x_{i}, y_{i}\right),\left(z_{i}, w_{i}\right) \in X_{i} \times X_{i}$,

$$
\left(x_{i}, y_{i}\right) \succsim^{*}\left(z_{i}, w_{i}\right) \Longleftrightarrow f_{i}\left(x_{i}\right)-f_{i}\left(y_{i}\right) \geq f_{i}\left(z_{i}\right)-f_{i}\left(w_{i}\right) .
$$

To prove this, it suffices to show that, for a nonnull $i \in N$, the following two claims are valid: for all $\left(x_{i}, y_{i}\right),\left(z_{i}, w_{i}\right) \in X_{i} \times X_{i}$,

(1) if $f_{i}\left(x_{i}\right)-f_{i}\left(y_{i}\right)>f_{i}\left(z_{i}\right)-f_{i}\left(w_{i}\right)$, then $\left(x_{i}, y_{i}\right) \succ^{*}\left(z_{i}, w_{i}\right)$;

(2) if $f_{i}\left(x_{i}\right)-f_{i}\left(y_{i}\right)=f_{i}\left(z_{i}\right)-f_{i}\left(w_{i}\right)$, then $\left(x_{i}, y_{i}\right) \sim^{*}\left(z_{i}, w_{i}\right)$.

To verify (1), suppose that $f_{i}\left(x_{i}\right)-f_{i}\left(y_{i}\right)>f_{i}\left(z_{i}\right)-f_{i}\left(w_{i}\right)$. We first assume that there are $\boldsymbol{a}, \boldsymbol{b} \in X$ such that

$$
f_{i}\left(x_{i}\right)-f_{i}\left(y_{i}\right) \geq \phi_{i}(\boldsymbol{a}, \boldsymbol{b}) \geq f_{i}\left(z_{i}\right)-f_{i}\left(w_{i}\right) .
$$

Then

$$
\begin{aligned}
& f_{i}\left(x_{i}\right)+\sum_{j \neq i} f_{j}\left(b_{j}\right) \geq f_{i}\left(y_{i}\right)+\sum_{j \neq i} f_{j}\left(a_{j}\right), \\
& f_{i}\left(w_{i}\right)+\sum_{j \neq i} f_{j}\left(a_{j}\right) \geq f_{i}\left(z_{i}\right)+\sum_{j \neq i} f_{j}\left(b_{j}\right),
\end{aligned}
$$

one of which must be strict. Therefore, either $x_{i} \boldsymbol{b} \succsim y_{i} \boldsymbol{a}$ and $w_{i} \boldsymbol{a} \succ z_{i} \boldsymbol{b}$, or $x_{i} \boldsymbol{b} \succ y_{i} \boldsymbol{a}$ and $w_{i} \boldsymbol{a} \succsim z_{i} \boldsymbol{b}$. If $x_{i} \boldsymbol{b} \succsim y_{i} \boldsymbol{a}, w_{i} \boldsymbol{a} \succ z_{i} \boldsymbol{b}$, and $\left(z_{i}, w_{i}\right) \succsim^{*}\left(x_{i}, y_{i}\right)$, then, by the consistency axiom, $z_{i} \boldsymbol{b} \succsim w_{i} \boldsymbol{a}$, a contradiction. Similarly, assume that $x_{i} \boldsymbol{b} \succ y_{i} \boldsymbol{a}, w_{i} \boldsymbol{a} \succsim z_{i} \boldsymbol{b}$, and $\left(z_{i}, w_{i}\right) \succsim^{*}\left(x_{i}, y_{i}\right)$. Then, by axiom EP2, $\left(y_{i}, x_{i}\right) \succsim^{*}\left(w_{i}, z_{i}\right)$. The consistency axiom implies that $y_{i} \boldsymbol{a} \succsim x_{i} \boldsymbol{b}$, a contradiction. Hence, $\left(x_{i}, y_{i}\right) \succ^{*}\left(z_{i}, w_{i}\right)$.

Next we assume that, for no $\boldsymbol{a}, \boldsymbol{b} \in X$,

$$
f_{i}\left(x_{i}\right)-f_{i}\left(y_{i}\right) \geq \phi_{i}(\boldsymbol{a}, \boldsymbol{b}) \geq f_{i}\left(z_{i}\right)-f_{i}\left(w_{i}\right) .
$$

Since $\phi_{i}(\boldsymbol{a}, \boldsymbol{a})=0$ for all $\boldsymbol{a} \in X$, we must have either $f_{i}\left(x_{i}\right)-f_{i}\left(y_{i}\right)>f_{i}\left(z_{i}\right)-f_{i}\left(w_{i}\right)>0$ or $0>f_{i}\left(x_{i}\right)-f_{i}\left(y_{i}\right)>f_{i}\left(z_{i}\right)-f_{i}\left(w_{i}\right)$. Assume $f_{i}\left(x_{i}\right)-f_{i}\left(y_{i}\right)>f_{i}\left(z_{i}\right)-f_{i}\left(w_{i}\right)>0$. For the other case the proof is similar. Since $f_{1}, \ldots, f_{n}$ are continuous, it is impossible to 
have that $\phi_{i}(\boldsymbol{a}, \boldsymbol{b})>f_{i}\left(x_{i}\right)-f_{i}\left(y_{i}\right)$ for some $\boldsymbol{a}, \boldsymbol{b} \in X$. To see this, it suffices to notice that $f_{i}\left(x_{i}\right)-f_{i}\left(y_{i}\right) \geq \phi_{i}(t \boldsymbol{a}+(1-t) \boldsymbol{b}, \boldsymbol{b}) \geq f_{i}\left(z_{i}\right)-f_{i}\left(w_{i}\right)$ for some $0<t<1$. Thus, for all $\boldsymbol{a}, \boldsymbol{b} \in X$,

$$
f_{i}\left(x_{i}\right)-f_{i}\left(y_{i}\right)>f_{i}\left(z_{i}\right)-f_{i}\left(w_{i}\right)>\phi_{i}(\boldsymbol{a}, \boldsymbol{b}) .
$$

Let

$$
\epsilon_{i}=\sup \left\{\phi_{i}(\boldsymbol{a}, \boldsymbol{b}): \boldsymbol{a}, \boldsymbol{b} \in X\right\}
$$

Then $\epsilon_{i}>0$ since some $j \neq i$ is nonnull. Take any positive integer $k$ such that

$$
\epsilon_{i}>\frac{f_{i}\left(z_{i}\right)-f_{i}\left(w_{i}\right)}{k}
$$

Hence, for some $\boldsymbol{a}^{\prime}, \boldsymbol{b}^{\prime} \in X$,

$$
\frac{f_{i}\left(x_{i}\right)-f_{i}\left(y_{i}\right)}{k}>\phi_{i}\left(\boldsymbol{a}^{\prime}, \boldsymbol{b}^{\prime}\right)>\frac{f_{i}\left(z_{i}\right)-f_{i}\left(w_{i}\right)}{k} .
$$

Since $f_{i}$ is continuous and strictly increasing, there are $c_{i}^{1}, \ldots, c_{i}^{k}, d_{i}^{1}, \ldots, d_{i}^{k} \in X_{i}$ such that, for $j=0,1, \ldots, k$,

$$
\begin{aligned}
f_{i}\left(c_{i}^{j}\right)-f_{i}\left(c_{i}^{j+1}\right) & =\frac{f_{i}\left(x_{i}\right)-f_{i}\left(y_{i}\right)}{k} \\
f_{i}\left(d_{i}^{j}\right)-f_{i}\left(d_{i}^{j+1}\right) & =\frac{f_{i}\left(z_{i}\right)-f_{i}\left(w_{i}\right)}{k}
\end{aligned}
$$

where $c_{i}^{0}=x_{i}, c_{i}^{k+1}=y_{i}, d_{i}^{0}=z_{i}$, and $d_{i}^{k+1}=w_{i}$. Thus, for $j=0,1, \ldots, k$,

$$
f_{i}\left(c_{i}^{j}\right)-f_{i}\left(c_{i}^{j+1}\right)>\phi_{i}\left(\boldsymbol{a}^{\prime}, \boldsymbol{b}^{\prime}\right)>f_{i}\left(d_{i}^{j}\right)-f_{i}\left(d_{i}^{j+1}\right) .
$$

It follows from the preceding paragraph that $\left(c_{i}^{j}, c_{i}^{j+1}\right) \succ^{*}\left(d_{i}^{j}, d_{i}^{j+1}\right)$ for $j=0,1, \ldots, k$, i.e.,

$$
v_{i}\left(c_{i}^{j}\right)-v_{i}\left(c_{i}^{j+1}\right)>v_{i}\left(d_{i}^{j}\right)-v_{i}\left(d_{i}^{j+1}\right) .
$$

Summation of these equalities gives $v_{i}\left(c_{i}^{0}\right)-v_{i}\left(c_{i}^{k+1}\right)>v_{i}\left(d_{i}^{0}\right)-v_{i}\left(d_{i}^{k+1}\right)$. Hence, $\left(x_{i}, y_{i}\right) \succ^{*}$ $\left(z_{i}, w_{i}\right)$.

To verify (2), suppose that $f_{i}\left(x_{i}\right)-f_{i}\left(y_{i}\right)=f_{i}\left(z_{i}\right)-f_{i}\left(w_{i}\right)$. Also, suppose that $f_{i}\left(x_{i}\right)-$ $f_{i}\left(y_{i}\right) \geq 0$. When $f_{i}\left(x_{i}\right)-f_{i}\left(y_{i}\right)<0$, the proof is similar. First, we assume that $f_{i}\left(x_{i}\right)-$ $f_{i}\left(y_{i}\right) \leq \phi_{i}\left(\boldsymbol{a}^{\prime}, \boldsymbol{b}^{\prime}\right)$ for some $\boldsymbol{a}^{\prime}, \boldsymbol{b}^{\prime} \in X$. Then, by continuity of $f_{i}, f_{i}\left(x_{i}\right)-f_{i}\left(y_{i}\right)=\phi_{i}\left(\boldsymbol{a}^{\prime \prime}, \boldsymbol{b}^{\prime \prime}\right)$ for some $\boldsymbol{a}^{\prime \prime}, \boldsymbol{b}^{\prime \prime} \in X$, so that $x_{i} \boldsymbol{b}^{\prime \prime} \sim y_{i} \boldsymbol{a}^{\prime \prime}$ and $z_{i} \boldsymbol{b}^{\prime \prime} \sim w_{i} \boldsymbol{a}^{\prime \prime}$. If $\left(x_{i}, y_{i}\right) \succ^{*}\left(z_{i}, w_{i}\right)$, then the consistency axiom implies $x_{i} \boldsymbol{b}^{\prime \prime} \succ y_{i} \boldsymbol{a}^{\prime \prime}$. If $\left(z_{i}, w_{i}\right) \succ^{*}\left(x_{i}, y_{i}\right)$, then similarly $z_{i} \boldsymbol{b}^{\prime \prime} \succ w_{i} \boldsymbol{a}^{\prime \prime}$. These are contradictions. Hence $\left(x_{i}, y_{i}\right) \sim^{*}\left(z_{i}, w_{i}\right)$.

Next, we assume that $f_{i}\left(x_{i}\right)-f_{i}\left(y_{i}\right)>\phi_{i}(\boldsymbol{a}, \boldsymbol{b})$ for all $\boldsymbol{a}, \boldsymbol{b} \in X$. Then $f_{i}\left(x_{i}\right)-f_{i}\left(y_{i}\right)>$ $\phi_{i}\left(\boldsymbol{a}^{\prime}, \boldsymbol{b}^{\prime}\right)>0$ for some $\boldsymbol{a}^{\prime}, \boldsymbol{b}^{\prime} \in X$. Thus, for some integer $k>0$, 


$$
\phi_{i}\left(\boldsymbol{a}^{\prime}, \boldsymbol{b}^{\prime}\right) \geq \frac{f_{i}\left(x_{i}\right)-f_{i}\left(y_{i}\right)}{k} .
$$

Therefore, by continuity of $f_{i}$, there are $c_{i}^{1}, \ldots, c_{i}^{k}, d_{i}^{1}, \ldots, d_{i}^{k} \in X_{i}$ such that, for $j=$ $0,1, \ldots, k$,

$$
f_{i}\left(c_{i}^{j}\right)-f_{i}\left(c_{i}^{j+1}\right)=f_{i}\left(d_{i}^{j}\right)-f_{i}\left(d_{i}^{j+1}\right)=\frac{f_{i}\left(x_{i}\right)-f_{i}\left(y_{i}\right)}{k},
$$

where $c_{i}^{0}=x_{i}, c_{i}^{k+1}=y_{i}, d_{i}^{0}=z_{i}$, and $d_{i}^{k+1}=w_{i}$. Note that

$$
\phi_{i}\left(\boldsymbol{a}^{\prime \prime}, \boldsymbol{b}^{\prime \prime}\right)=\frac{f_{i}\left(x_{i}\right)-f_{i}\left(y_{i}\right)}{k} \text { for some } \boldsymbol{a}^{\prime \prime}, \boldsymbol{b}^{\prime \prime} \in X .
$$

Thus it follows from the preceding paragraph that $\left(c_{i}^{j}, c_{i}^{j+1}\right) \sim^{*}\left(d_{i}^{j}, d_{i}^{j+1}\right)$ for $j=0,1, \ldots, k$, so that

$$
v_{i}\left(c_{i}^{j}\right)-v_{i}\left(c_{i}^{j+1}\right)=v_{i}\left(d_{i}^{j}\right)-v_{i}\left(d_{i}^{j+1}\right) .
$$

Adding these equalities, we obtain $v_{i}\left(c_{i}^{0}\right)-v_{i}\left(c_{i}^{k+1}\right)=v_{i}\left(d_{i}^{0}\right)-v_{i}\left(d_{i}^{k+1}\right)$, which implies $\left(x_{i}, y_{i}\right) \sim^{*}\left(z_{i}, w_{i}\right)$. This completes the proof of the claims (1) and (2).

If $i$ is nonnull, then, on $X_{i} \times X_{i}$, we have

$$
\begin{aligned}
\left(x_{i}, y_{i}\right) \succsim^{*}\left(z_{i}, w_{i}\right) & \Longleftrightarrow f_{i}\left(x_{i}\right)-f_{i}\left(y_{i}\right) \geq f_{i}\left(z_{i}\right)-f_{i}\left(w_{i}\right) \\
& \Longleftrightarrow v_{i}\left(x_{i}\right)-v_{i}\left(y_{i}\right) \geq v_{i}\left(z_{i}\right)-v_{i}\left(w_{i}\right)
\end{aligned}
$$

Therefore, $f_{i}=\alpha_{i} v_{i}+\beta_{i}$ for some numbers $\alpha_{i}>0$ and $\beta_{i}$. By assumption, $N^{*}=\{1, \ldots, m\}$ is the set of all nunnull states. Thus $\sum_{i=1}^{m} \alpha_{i}>0$. We define $p_{i}=\alpha_{i} / \sum_{k=1}^{m} \alpha_{k}$ for all $i \in N^{*}$. Hence, $\boldsymbol{p}=\left(p_{1}, \ldots, p_{m}\right)$ is a unique positive probability vector in $\mathbb{R}^{m}$ and, for all $\boldsymbol{x}, \boldsymbol{y} \in X$,

$$
\begin{aligned}
\boldsymbol{x} \succsim \boldsymbol{y} & \Longleftrightarrow \sum_{i=1}^{n} f_{i}\left(x_{i}\right) \geq \sum_{i=1}^{n} f_{i}\left(y_{i}\right) \\
& \Longleftrightarrow \sum_{i=1}^{m} f_{i}\left(x_{i}\right) \geq \sum_{i=1}^{m} f_{i}\left(y_{i}\right) \\
& \Longleftrightarrow \sum_{i=1}^{m}\left(\alpha_{i} v_{i}\left(x_{i}\right)+\beta_{i}\right) \geq \sum_{i=1}^{m}\left(\alpha_{i} v_{i}\left(y_{i}\right)+\beta_{i}\right) \\
& \Longleftrightarrow \sum_{i=1}^{m} p_{i} v_{i}\left(x_{i}\right) \geq \sum_{i=1}^{m} p_{i} v_{i}\left(y_{i}\right) .
\end{aligned}
$$

This completes the proof. 


\section{Conclusion}

In the subjective expected utility paradigm, this paper considered two primitive preference relations, i.e. ex ante preference and ex post strength-of-preference relations, whose numerical representations are well known in the literature. The main contribution of the paper was to discover a consistency condition between ex ante preference and ex post strength-ofpreference relations which can separate utility and unique subjective state probabilities.

\section{Acknowledgements}

The author acknowledges useful comments of two anonymous referees to improve readability of the paper. In particular, it is appreciated that the present weaker form of the consistency axiom was suggested by one of the referees.

\section{References}

Anscombe, F.J. and Aumann, R.J. (1963) A definition of subjective probability. Annals of Mathematical Statistics 34, 199-205.

Butler, D., Isoni, A., Loomes, G., and Navarro-Martinez, D., On the measurement of strength of preference in units of money, Economic Record 90, Special Issue, 1-15, 2014.

Debreu, G. (1954) Representation of a preference ordering by a numerical function. In R.M. Thrall, C.H. Coombs, and R.L. Davis (eds.) Decision Processes, Wiley, New York.

Drèze, J.H. and Rustichini, A. (2004) State-dependent utility and decision theory. In S. Barbera, P. Hammond, and C. Seidl (eds.) Handbook of Utility Theory, Volume 2: Extensions, Springer.

Farquhar, P.H. \& Keller, L.R., (1989) Preference intensity measurement, Annals of Operations Research 19, 205-217.

Krantz, D.H., Luce, R.D., Suppes, P. and Tversky, A. (1971) Foundations of Measurement, Volume 1: Additive and Polynomial Representations. Academic Press.

Sarin, R.K. (1982) Strength of preference and risky choice, Operations Research 30(5) 982-997.

Savage, L.J. (1954) The Foundations of Statistics. Wiley, New York.

von Winterfeldt, D. and Edwards, W. (1986) Decision Analysis and Behavioral Research, Cambridge University Press, Cambridge.

Wakker, P.P. (1989) Additive Representations of Preferences: A New Foundation of Decision Analysis. Dordrecht: Kluwer Academic Publishers.

Wakker, P.P. and Zank, H. (1999) State dependent expected utility for Savage's state space. Mathematics of Operations Research 24, 8-34. 